\title{
LA-UR-02-0521
}

Approved for public release; distribution is unlimited.

Los Alamos National Laboratory

AIAA 2002-1570

Submitted to: $43^{\text {rd }}$ Structures, Structural Dynamics and

Materials Conference

Denver CO

April 22-25, 2002

\section{LINKING PROBABILITY THEORY AND FUZZY SETS-A STUDY IN UNCERTAINTY ASSESSMENT}

\author{
Jane M. Booker and Thomas R. Bement (posthumously) \\ Los Alamos National Laboratory \\ Los Alamos, New Mexico \\ Kimberly F. Sellers* \\ Carnegie Mellon University \\ Pittsburgh, Pennsylvania \\ Nozer D. Singpurwalla ${ }^{\phi}$ \\ The George Washington University \\ Washington, D.C.
}

\begin{abstract}
Uncertainties enter into a complex problem from many sources: variability, errors, and lack of knowledge. A fundamental question arises in how to characterize the various kinds of uncertainty and then combine within a problem such as the verification and validation of a computer model, reliability of a dynamic system, or a complex decision problem. Our aim is to explore how probability theory and fuzzy set theory can be made to work in concert, so that uncertainty of outcomes and imprecision can be treated in a unified and coherent manner. Both the theoretical and application of a linkage between the two theories will be presented. An example from a reliability application will illustrate how the linkage between the theories is accomplished through the use of Bayes Theorem and the probability of fuzzy sets.
\end{abstract}

\section{INTRODUCTION}

To many, the term uncertainty means an absence of knowledge. To some variability or variation is considered distinct from uncertainty. To others the term embodies multiple sources including variability and errors. Regardless of the source, a fundamental question arises in how to characterize the various kinds of uncertainty and then combine them for a given problem in light of decision-making. Examples of such complex problems include computer model verification and validation, and reliability prediction applications with little or sparse data.

Broadly speaking, uncertainties can be classified into one of two sources reflecting the meaning and nature of uncertainty-aleatory or epistemic. Aleatory uncertainty is found in physical and natural variation, which cannot be reduced given new

\footnotetext{
*Visiting Assistant Professor, Department of Statistics

Professor of Operations Research and of Statistics and Distinguished Research Professor

This paper is declared the work of the U.S. Government and is not subject to copyright protection in the United States.
} 
information, data, or knowledge. Epistemic uncertainty is from the lack of knowledge and can be reduced by increasing sample size or gaining new information. Some propose a third category, error, which could be either aleatory or epistemic, and refers to errors, mistakes, and numerical miscalculation. It is interesting to note the difficulty in classifying the types of uncertainty into crisp categories. Fuzzy sets are designed to address this kind of uncertainty in classification.

Since Zadeh's introduction of fuzzy set theory in 1965, probability and statistics are sometimes considered inadequate for dealing with particular kinds of uncertainty (even if data are available), and probability models only the type of uncertainty arising from the unknown outcome of an experiment. Since it is quite possible that both unknown outcomes and imprecision of classification can be present in the same problem, Zadeh has also claimed that "probability must be used in concert with fuzzy logic to enhance its effectiveness. In this perspective, probability theory and fuzzy logic are complementary rather than competitive." Agreeing with this philosophy, we explore how probability theory and fuzzy set theory can be made to work in concert, so that uncertainty of outcomes and imprecision can be treated in a unified and coherent manner.

Both the theory and application of a linkage between the two constructs will be presented in the sections below. The application is taken from the PREDICT information integration methodology ${ }^{3}$, where traditional reliability data is sparse but decisions on performance are nonetheless required. In particular, the example illustrates how the linkage between the theories is accomplished through the use of Bayes Theorem and the development of the probability measure of fuzzy sets.

\section{TWO THEORIES FOR UNCERTAINTY}

\section{Probability Theory}

Probability theory can be described as providing a calculus (an algebra) for determining the uncertainty of outcomes of an experiment or event, $E$. Let $\Omega$ represent the set of all possible outcomes of $E$. The theory does not tell us how to specify $\Omega$, but $\Omega$ may be countable and $\Omega^{C}=\varnothing$. Let $F$ denote the set of all subsets belonging to $\Omega$. Define subsets $A$ and $B$ such that $A, B \in F$, and then
$(A \cup B) \in F$ and $(A \cap B) \in F$. These subsets are well defined (or crisp). There is no ambiguity in determining whether an outcome of $E$ belongs to $A$ or it complement, $A^{C}$.

Because $E$ is going to be performed, we are uncertain about the outcome of $E$, say $\omega$. Let $P(A)$ describe our uncertainty about the outcome, where $0 \leq P(A) \leq 1$, and $P(A)$ represents our bet that $\omega \in$ A. $P(A)$ is the probability measure of the set $A$ and is known as the probability of event $A$. The bet is two-sided on the occurrence or non-occurrence of event $A$, and it will be unambiguously settled when $E$ is performed and $\omega$ is observed.

Probability theory does not tell us how to arrive upon a $P(A)$, nor how to interpret $P(A)$. There are many different interpretations of probability ${ }^{4}$. The willingness to bet is one, referred to as the personalistic or subjective interpretation of probability.

The calculus of probability dictates how this kind of uncertainty combines or coheres for two or more events. Similarly, we specify $P(B)$ as a quantification of uncertainty of another event, $B$, when $E$ is performed, for $\omega \in B$. Then the basic axioms of probability are:

$$
\begin{gathered}
0 \leq P(\mathrm{~A}) \leq 1 \\
P(A \cup B)=P(A)+P(B)-P(A \cap B) \\
P(\mathrm{~A} \cap \mathrm{B})=P(A \mid B) P(B),
\end{gathered}
$$

where $P(A \mid B)$ is the conditional probability of $A$ were $B$ to occur.

Bayes Theorem is an important development of conditional probabilities. If $A$ is broken down into a sequence of events, $A_{1}, A_{2}, \ldots$, then the discrete probability form of the theorem is:

$$
P\left(A_{i} \mid B\right)=\left[P\left(B \mid A_{i}\right) P\left(A_{i}\right)\right] / P(B), j=1,2, \ldots,
$$
where $P(B)=\Sigma_{k} P\left(B \mid A_{k}\right) P\left(A_{k}\right)$. Correspondingly, the continuous form using probability density functions (pdf), $g$ and $f$, is:

$$
g(\theta \mid x)=[f(x \mid \theta) g(\theta)] / \int f(x \mid \theta) g(\theta) \mathrm{d} \theta,
$$

where $x$ is the random variable representing the data for a given value of parameter, $\theta$. The pdf on the left is the posterior distribution obtained by combining the function, $f$ (for the data) with the prior probability density function for $\theta$. The function $f$ can be replaced by the likelihood function, which is not a pdf. As will be seen later, the use of the likelihood function and Bayes theorem provide an important link between probability and fuzzy set theories. 


\section{Fuzzy Set Theory and Membership Functions}

Fuzzy set theory is useful in handling the kind of vague uncertainty that can be associated with classifying an event into a set. Unlike probability theory, which demands that any outcome $\omega$ belong to set $A$ or to $A^{C}$ and not both, fuzzy set theory permits such joint membership. The degree of membership belonging to any set is specified using a membership function. A short example illustrates:

Let $X$ denote the set of integers between zero and ten, inclusive: $X=\{0,1,2, \ldots, 10\}$. Suppose we are interested in a subset $A^{*}$ of $X$, where $A^{*}$ contains all the medium integers of $X$ :

$A^{*}=\{x ; x \in X$ and $x$ is medium $\}$. To specify $A^{*}$, the term medium integer must be defined. Most would consider 5 as medium, but what about 7? Our uncertainty (or vagueness) about what constitutes a medium integer is what makes $A^{*}$ a fuzzy set, and such sets occur in our everyday use (or natural language). The uncertainty of classification arises because the boundaries of $A^{*}$ are not crisp. The integer 7 might have some membership (belonging) in $A^{*}$, and yet also have some degree of membership in $A^{*}$.

A membership function, $m_{A^{*}}(X)$ can quantify this form of vagueness based on the degree of membership. Specifically, $m_{A^{*}}(x)$ is a number (by convention, between 0 and 1) that reflects, in the subjective view of an assessor, the extent to which $x \in A^{*}$. The assessor assigns to each $x \in X$ a number, $m_{A^{*}}(x)$, and this is done for all subsets of the type $A^{*}$ that are of interest.

As with probability, membership functions can be combined from two or more fuzzy sets using the following axioms:

$$
\begin{aligned}
m_{A^{*} \cup B^{*}}(x) & =\max \left[m_{A^{*}}(x), m_{B^{*}}(x)\right] \\
m_{A^{*} \cap B^{*}}(x) & =\min \left[m_{A^{*}}(x), m_{B^{*}}(x)\right] \\
m_{A^{*} C}(x) & =1-m_{A^{*}}(x) \\
\text { If } m_{A^{*}}(x) & =m_{B^{*}}(x), \text { then } A^{*}=B^{*} \\
\text { If } m_{A^{*}}(x) & \leq m_{B^{*}}(x), \text { then } A^{*} \subseteq B^{*}
\end{aligned}
$$

\section{LINKAGE BETWEEN THE THEORIES}

Four issues are important involving the interpretation of membership functions relative to probability theory. First, just as probability theory does not tell how to specify $P(A)$, fuzzy set theory does not tell how to specify $m_{A^{*}}(x)$. Second, while probability axiom (1) bounds $P(A), m_{A^{*}}(x)$ has no such bounds. In most applications, it is simply a convenience for $m_{A^{*}}(x) \in[0,1]$. Third, while $P(A)$ can be interpreted as a two-sided bet, $m_{A^{*}}(x)$ reflects an assessor's view of the extent to which $x \in A^{*}$. Finally, it is not a requirement that the sum over $x$ of all $m_{A^{*}}(x)$ equal one, precluding $m_{A^{*}}(x)$ as being interpreted as a probability.

Noting that $m_{A^{*}}(x)$, as a function of $x$, reflects the extent to which $x \in A^{*}$, it is an indicator of how likely it is that $x \in A^{*}$. One interpretation of $m_{A^{*}}(x)$ is as the likelihood of $x$ for a fixed (specified) $A^{*}$. A likelihood function is not a probability. In statistical inference, it is the relative degree of support that an observation provides to several hypotheses. Specifying the likelihood is also a subjective process, consistent with membership function definition and the interpretation of probability.

As noted below Equation (5), likelihoods are also part of Bayes Theorem. If membership functions can be interpreted as likelihoods, then Bayes Theorem provides a valuable link from fuzzy sets back into probability theory.

Related to this argument is the determination of the probability measure of fuzzy sets. Again, membership functions provide an important linkage between the two theories, and again the purely subjectivist interpretation will be important.

We begin with a subjective analyst (e.g., a design engineer), $D$, who wants to determine the probability of a fuzzy set, $A^{*}$,

$$
P_{D}\left(A^{*}\right)=P_{D}\left(X \in A^{*}\right)
$$

where $X$ denotes the uncertain outcome of an experiment, $E$, and the subscript denotes $D$ 's personal probability. Because fuzzy sets are involved, $D$ now has to contend with two types of uncertainty: the outcome of the experiment $X=x$ and the membership of $x$ in $A^{*}$. Using probability theory, $D$ specifies two probabilities for these uncertainties:

- $P_{D}(x), D$ 's prior probability that $x$ is the outcome of $E$ (an aleatory uncertainty), and

- $P_{D}\left(X \in A^{\dagger}\right)$, D's prior probability that the outcome $x$ belongs to $A$ (an epistemic uncertainty).

Determining the first probability is straightforward using $D$ 's knowledge about the experiment. The question becomes how $D$ determines the latter probability. Perfect knowledge (or God) knows exactly how to classify $x$ with perfect precision. While $D$ will never know this, he/she does have some partial knowledge about this classification, albeit, imprecise, which determines $P_{D}\left(X \in A^{*}\right)$. The law of total probability dictates how these two probabilities can be combined and interpreted:

$$
P_{D}\left(A^{*}\right)=P_{D}\left(X \in A^{*}\right)=\Sigma_{x} P_{D}\left(X \in A^{*} \mid X=x\right) \cdot P_{D}(x) .
$$


While $D$ can assess these probabilities, suppose he/she consults another expert, say $Z$ (in honor of Zadeh), whose knowledge is in the form of a membership function. $D$ needs to update equation (12) in light of this new information. Appealing to the law of total probability once more, and assuming $X$ is generated independently of $m_{A^{*}}(X), D$ gets:

$$
P_{D}\left(X \in A^{*} \mid m_{A^{*}}(x)\right)=\Sigma_{x} P_{D}\left(x \in A^{*} \mid m_{A^{*}}(x)\right) \cdot P_{D}(x) .
$$

The middle term in (13) can be evaluated using Bayes Theorem, written in the form of probabilities as

$$
P_{D}\left(x \in A^{*} \mid m_{A^{*}}(x)\right) \propto P_{D}\left(m_{A^{*}}(x) \mid x \in A^{*}\right) \cdot P_{D}\left(x \in A^{*}\right) .
$$

The middle term in (14) is actually the likelihood and, in this case, it corresponds to the membership function. Combining Equations (13) and (14), we establish the fundamental relationship between probability and membership functions, including the probability measure of fuzzy sets as:

$$
P_{D}\left(X \in A^{*} ; m_{A^{*}}(x)\right) \propto \Sigma_{x} m_{A^{*}}(x) \cdot P_{D}\left(x \in A^{*}\right) \cdot P_{D}(x)
$$

A more complete mathematical and interpretive development is available ${ }^{5}$.

\section{RELIABIILITY EXAMPLE}

A short example in reliability demonstrates the combining of two different kinds of uncertainty using equation (15). Reliability is the probability that the system will successfully perform its function for a given period of time and given specifications. Because reliability is a probability, the solution is in the space of probability theory, consistent with development of Equation (15).

Assume we have a concept design for a new automotive system, like a fuel injector. Many of the components are also new designs, but may be similar to ones used in the past, implying that partial knowledge exists. Yet the designer, $D$, wants to estimate reliability based upon whatever information is currently available before building prototypes or implementing expensive test programs. $D$ knows that knowledge, expertise, historical experience, and even information from vendors and suppliers of parts is valuable and, when properly elicited and quantified, can provide the desired reliability estimate under uncertainty. The information integration methodology, PREDICT (Performance and Reliability Evaluation with Diverse Information Combination and Tracking), provides the tools for such a daunting task ${ }^{3}$. Included among those tools are the methods for formally eliciting and analyzing the expert knowledge that exists within the experience base of the design experts and suppliers ${ }^{6}$.

Consider a system, $\underline{S}$, with three components $\underline{A}$, $\underline{B}, \underline{C}$, and one manufacturing process, $\underline{M P}$, with all items operating in a series reliability arrangement as shown in Figure $1 . \quad \underline{A}$ and $\underline{B}$ constitute subsystem $\mathrm{SS}$. This initial reliability logic flow diagram might be the result of $D$ 's first concept design for the system.

$D$ might decide upon a two-parameter Weibull model to reflect the reliability, representing both the components and the manufacturing process. The Weibull model gives a time dependent estimate of reliability $(R)$ :

$$
R(t)=\exp \left(-\lambda t^{\beta}\right)
$$

Having a time dependent model is useful for predicting reliability at strategic time periods for warranty and regulatory purposes (e.g., EPA regulations). Typical periods of interest are one and three years or 100,000 miles. It is important to note here that the conversion of miles to time is another source of epistemic uncertainty. The conversion can be accomplished using a probability density function to represent the uncertainty associated with how long it takes for various owners to accumulate 100,000 miles.

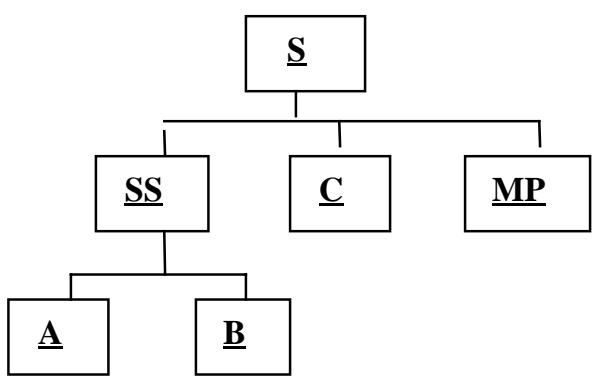

Figure 1. Simple system reliability logic flow diagram

Because the system is a new concept design, test data is lacking for its components and process. However, valuable information exists in the form of the expert knowledge of its designer, $D$. Using formal expert elicitation methods ${ }^{6}, D$ identifies the values and uncertainties for the two parameters, $\beta$ (the slope) and $\lambda$ (the failure rate per scaled unit of time) for each component and process. The epistemic uncertainties in these parameters are characterized by probability density functions and translate into uncertainties for the reliabilities through Equation (16). 
Each component and the MP are considered in series; therefore the reliability of $\underline{S}$ is the product of those individual reliabilities. The uncertainty distribution for the system reliability at 3 years (36 months) is then calculated from the individual uncertainty distributions using Monte Carlo simulation.

Upon examining the reliability uncertainty distribution for the system, $D$ realizes that improvements are necessary to make the reliability acceptable to the design community. By examining the uncertainty distributions that comprise the system, component $\underline{B}$ is seen to have the worst reliability, contributing the most to the over all poor system performance; therefore $D$ seeks additional knowledge about this component to combine with the prior probabilities that he/she has already provided.

$D$ consults the supplier of component $\underline{B}$ to see if any additional information about its reliability can be obtained before considering a test program to obtain more data. However, the supplier's information is not in terms of the Weibull probability-based model. Instead, the supplier $(Z)$ has membership functions for the reliability assessment of $\underline{B}$, shown in Figure 2, with descriptions in Table 1.

Table 1. Zs fuzzy set reliability assessment

$\begin{array}{cl}\text { Fuzzy Set } & \text { Linguistic Description } \\ A^{*} & \text { Reliability is excellent } \\ B^{*} & \text { Reliability is nominal } \\ C^{*} & \text { Reliability is poor } \\ D^{*} & \text { Reliability is unacceptable } \\ E^{*} & \text { Reliability is ridiculous }\end{array}$

Upon examination of the information from $Z, D$ now asks about the probability that the reliability will be in fuzzy sets $A^{*}$ or $B^{*}$, taking advantage of Equation (15) for combining the membership function information with $D$ 's prior probabilistic assessment from the reliability analysis as summarized in Table 2.

Table 2. $P_{D}(x)$, D's prior assessment of the reliability for $\underline{B}$

$\begin{array}{cc}\boldsymbol{x} & \boldsymbol{P}_{\boldsymbol{D}}(\boldsymbol{x}) \\ 0 & 0 \\ 0.25 & 0.0001 \\ 0.5 & 0.005 \\ 0.7 & 0.05 \\ 0.8 & 0.25 \\ 0.85 & 0.20 \\ 0.9 & 0.45 \\ 0.925 & 0.50 \\ 0.97 & 0.75 \\ 0.98 & 0.90 \\ 0.99 & 0.95 \\ 0.998 & 0.99 \\ 0.999 & 0.9999\end{array}$

To update component $\underline{B}$ in light of the new information from $Z$ according to Equation (15), $D$ must also assess the probabilities that values of $x$ belong to each of the five fuzzy sets. These assessments are shown in Table 3.

Multiplying D's probability values in Tables 2 and 3 with $Z$ s membership function values in Figure 2,

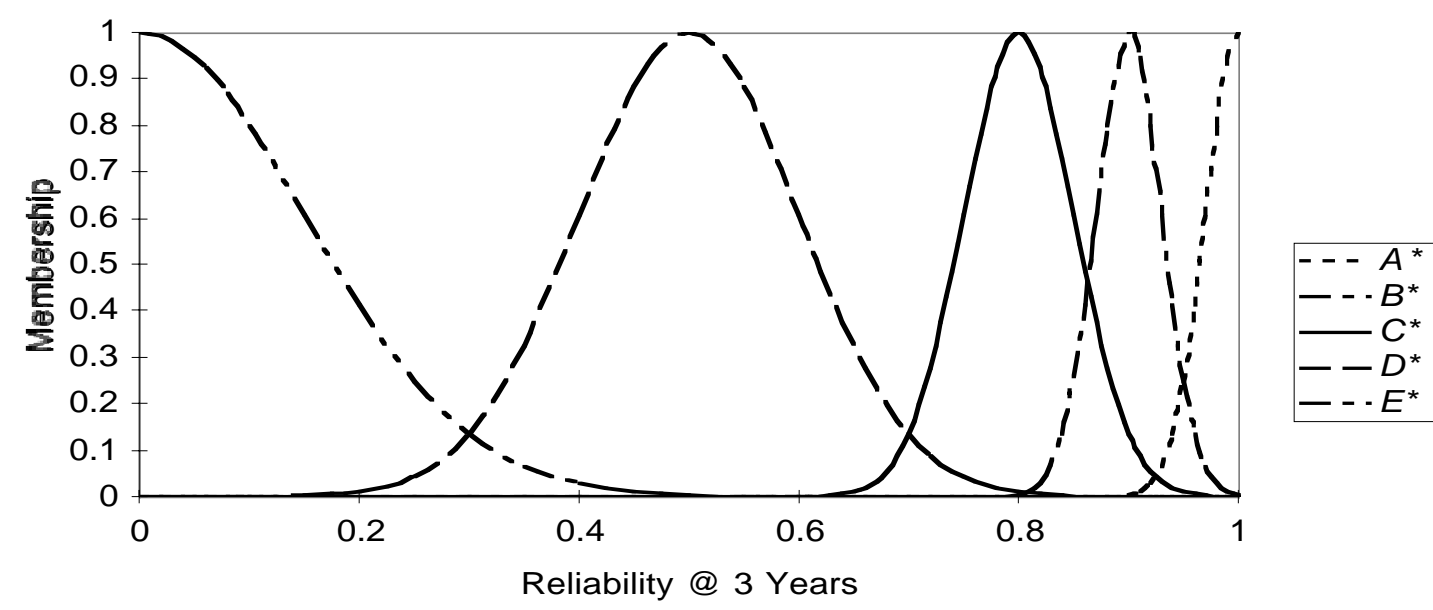

Figure 2. Reliability membership functions for component $\underline{B}$. 
and then summing over all values of $x$ in Equation (15) gives the desired probability for each of the five fuzzy sets. However, these results are not normalized. After normalizing for the five possible sets (in Table 4), $D$ obtains the estimates for the reliability of component $\underline{B}$ based on the inclusion of both kinds of uncertainty and all the available information.

Table 3. D's assessment of $P_{D}(x \in$ fuzzy set)

$\begin{array}{cccccc}\boldsymbol{x} & \boldsymbol{P}_{\boldsymbol{D}}\left(\boldsymbol{x} \in A^{\boldsymbol{*}}\right) & \boldsymbol{P}_{\boldsymbol{D}}\left(\boldsymbol{x} \in B^{*}\right) & \boldsymbol{P}_{\boldsymbol{D}}\left(\boldsymbol{x} \in C^{*}\right) & \boldsymbol{P}_{\boldsymbol{D}}\left(\boldsymbol{x} \in \boldsymbol{D}^{*}\right) & \boldsymbol{P}_{\boldsymbol{D}}\left(\boldsymbol{x} \in E^{*}\right) \\ 0 & 0 & 0 & 0 & 0 & 0.91 \\ 0.25 & 0 & 0 & 0.025 & 0.05 & 0.09 \\ 0.5 & 0 & 0 & 0.11 & 0.495 & 0 \\ 0.7 & 0 & 0 & 0.18 & 0.4 & 0 \\ 0.8 & 0 & 0.005 & 0.23 & 0.05 & 0 \\ 0.85 & 0.002 & 0.20 & 0.21 & 0.005 & 0 \\ 0.9 & 0.038 & 0.225 & 0.18 & 0 & 0 \\ 0.925 & 0.14 & 0.21 & 0.06 & 0 & 0 \\ 0.97 & 0.15 & 0.18 & 0.005 & 0 & 0 \\ 0.98 & 0.16 & 0.16 & 0 & 0 & 0 \\ 0.99 & 0.17 & 0.02 & 0 & 0 & 0 \\ 0.998 & 0.17 & 0 & 0 & 0 & 0 \\ 0.999 & 0.17 & 0 & 0 & 0 & 0\end{array}$

Table 4 indicates that the probability of "excellent" reliability is 0.74 and that of "nominal" reliability is 0.17 . $D$ was hoping that those two fuzzy sets would produce a combined reliability value high enough to drive the system reliability to greater than 0.96, to meet requirements and avoid a test or prototype program. While falling a bit short of this goal, $D$ examines the other components to determine if additional information on those components improves the reliability for $\underline{\mathrm{S}}$. In particular, another component has a large uncertainty, whose reduction may be achieved by investigating the existence of other information.

Table 4. Normalized $P_{D}(x \in$ fuzzy set $)$

$\begin{array}{ll}\boldsymbol{P}_{D}\left(x \in A^{*}\right) & 0.740 \\ \boldsymbol{P}_{D}\left(x \in \boldsymbol{B}^{*}\right) & 0.172 \\ \boldsymbol{P}_{D}\left(x \in C^{*}\right) & 0.085 \\ \boldsymbol{P}_{D}\left(x \in \boldsymbol{D}^{*}\right) & 0.003 \\ \boldsymbol{P}_{D}\left(x \in E^{*}\right) & 0.000\end{array}$

Once all additional information and knowledge is acquired and included into the reliability analysis for $\underline{\mathrm{S}}$, then more expensive options such as a test or prototype program can be considered. Upon considering such expensive options, $D$ can estimate the reliability from potential outcomes of tests by continuing to update the reliability calculations. For example, $D$ can ask: "What if we build 38 parts, test them and they all pass, what impact would this have on reliability?" Methods for this kind of updating calculation (before actually implementing the test program) can be found in the PREDICT methodology ${ }^{3}$. If the reliability is not sufficiently improved nor the uncertainty reduced with such a test result, then $D$ would reconsider proposing that expensive program, and he/she would pose other "what if" questions, including design changes to determine the courses of action necessary to achieve the reliability goal.

\section{CONCLUDING REMARKS}

The reliability example serves to illustrate how different types of uncertainty can be accommodated using two mathematical theories: probability and fuzzy set, and how these two theories can work in concert. Other alternative theories exist ${ }^{7}$; however, linkages between them are a matter of continued research. Complex decision problems such as verification and validation of computational and simulation models are replete with uncertainties (aleatory and epistemic) and errors in:

- experimental data (e.g., outcomes of experiments and measurement errors),

- models and their parameters,

- numerical and computational methods.

Understanding, estimating and analytically handling these may require the use of additional theories for uncertainties, and will require linkages between theories so that all can be combined coherently within the same problem. This paper is only the first step along that long and difficult journey.

\section{REFERENCES}

1. Zadeh. L., "Fuzzy Sets," Information and Control, 8, 338-353, 1965.

2. Zadeh. L., "Discussion: Probability Theory and Fuzzy Logic Are Complementary Rather Than Competitive," Technometrics, 37, 271-276, 1995

3. Booker, J., Bement, T., Meyer, M., Kerscher, W. "PREDICT: A New Approach to Product Development and Lifetime Assessment Using Information Integration Technology," to appear Handbook of Statistics: Statistics in Industry (Rao and Khattree, eds.), 2002. 
4. Bement, T. Booker, J, Keller-McNulty, S., Singpurwalla, N., "Testing the Untestable; Reliability in the $21^{\text {st }}$ Century," to appear IEEE Transactions on Reliability, 2002.

5. Singpurwalla, N, Booker, J. "Membership Functions and Probability Measures of Fuzzy Sets," Los Alamos National Laboratory report, LA-UR-020032, to be submitted to JASA, 2002.

6. Meyer, M., Booker, J. Eliciting and Analyzing Expert Judgment: A Practical Guide, Society of Industrial and Applied Mathematics (SIAM), Philadelphia, PA, 2001.

7. Oberkampf, W, Helton, J, Sentz, K, "Mathematical Representation of Uncertainty," NonDeterministic Approaches Forum, April, 2001, AIAA Proceedings, Reston, VA, 2001. 